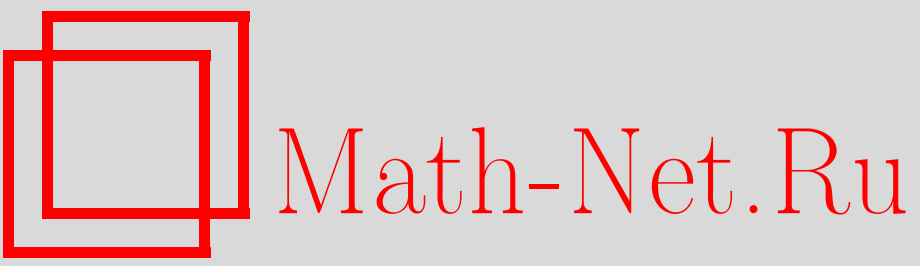

В. В. Белокуров, Ю. П. Соловьев, Е. Т. Шавгулидзе, Метод приближенного вычисления континуальных интегралов, использующий теорию возмущений со сходящимися рядами. II. Евклидова квантовая теория поля, ТМФ, 1996, том 109, номер 1, 60-69

DOI: https://doi.org/10.4213/tmf1211

Использование Общероссийского математического портала Math-Net.Ru подразумевает, что вы прочитали и согласны с пользовательским соглашением

http://www.mathnet.ru/rus/agreement

Параметры загрузки:

IP : 52.205.19.152

26 апреля 2023 г., 14:37:41 


\author{
ТЕОРЕТИЧЕСКАЯ \\ И МАТЕМАТИЧЕСКАЯ \\ ФИЗИКА \\ Том 109, № 1 \\ октябрь, 1996
}

В.В. Белокуров*, Ю. П. Соловьев* , Е. Т. Шавгулидзе*

\title{
МЕТОД ПРИБЛИЖЕННОГО ВЫЧИСЛЕНИЯ КОНТИНУАЛЬНЫХ ИНТЕГРАЛОВ, ИСПОЛЬЗУЮЩИЙ ТЕОРИЮ ВОЗМУЩЕНИЙ СО СХОДЯШИМИСЯ РЯДАМИ. \\ II. ЕВКЛИДОВА КВАНТОВАЯ ТЕОРИЯ ПОЛЯ
}

Дается обоснование предложенного в предыдущей работе [1] метода приближенного вычисления континуальных интегралов для интегралов в гильбертовом пространстве в случае, когда гауссова мера задается некоторым ядерным оператором.

1. Как известно, полные функции Грина в евклидовой квантовой теории поля могут быть представлены в виде континуальных интегралов по полю $\phi$ типа

$$
\int \phi\left(x_{1}\right) \ldots \phi\left(x_{n}\right) \exp \left\{-S_{0}[\phi]-S_{\text {int }}[g, \phi]\right\} d \phi
$$

в показатель экспоненты которых входит действие свободной теории $S_{0}[\phi]$, квадратичное по полю $\phi$, и член взаимодействия $S_{\mathrm{int}}[g, \phi]$, содержащий степени полевой функции старше второй.

В этом случае, однако, известны значения интегралов, как правило, только квазигауссова типа

$$
\int \mathcal{P}(\phi) \exp \left\{-S_{0}[\phi]\right\} d \phi
$$

$(\mathcal{P}(\phi)$ - произвольный полином от $\phi)$. Для вычисления же континуальных интегралов вида (1) их разлагают в ряды по степеням $g$. При этом каждый член ряда является интегралом квазигауссова типа (2).

Важным свойством построенной таким образом теории возмушений оказывается то, что получаемые ряды расходятся и имеют смысл лишь асимптотических разложений при малых $g$ [2]. Это является серьезным препятствием к вычислению континуальных интегралов при больших $g$. Более того, даже и в случае малых $g$, когда разложения справедливы, невозможно определить точность проведенного вычисления.

${ }^{*}$ Московский государственный университет 
В предыдушей статье [1] мы предложили метод приближенного выгисления континуальных интегралов, в котором используются только сходящиеся ряды. В основе метода лежит некоторое интегральное представление (нелинейное преобразование Фурье) стоящего в подынтегральном выражении множителя $\exp (-g P(x))(P(x)$ - однородньй полином степени $2 m$ ). При этом функция

$$
\exp \left(i g^{\frac{1}{m}} \rho P^{\frac{1}{m}}(x)\right)
$$

с помощью которой совершается данное преобразование Фурье, раскладывается в ряд. Кроме того, для возможности последуюшей перестановки операций суммирования и интегрирования в этом интегральном представлении выбирается конечная область интегрирования (появляется зависимость от дополнительного параметра $R$ ). Таким образом, в случае взаимодействия $g \phi^{2 m}$ получаются абсолютно сходяшиеся ряды, которые представляют собой разложения по степеням параметра $g^{\frac{1}{m}}$.

Коэффициенты при целых степенях $g^{n}$ для малых показателей $n(n \leq 4)$ практически совпадают с соответствуюшими коэффициентами в асимптотическом разложении, но затем начинают все более отличаться, стремясь к нулю при $n \rightarrow \infty$ (ряд сходящийся!).

В статье [1] метод построения сходящейся теории возмушений был сформулирован на примере конечномерных интегралов - "нульмерных" аналогов континуальных интегралов, возникаюших в квантовой теории поля. В настоящей работе мы покажем, как этот метод работает в случае континуальных интегралов в гильбертовом пространстве.

2. Прежде всего напомним некоторые определения.

Пусть $H$ - сепарабельное гильбертово пространство и $T: H \rightarrow H$ - самосопряженный положительно определенный ядерньй оператор. Это означает, что в $H$ существует такой ортонормированный базис $e_{1}, \ldots, e_{n}, \ldots$, что

$$
T e_{n}=\lambda_{n} e_{n}, \quad \lambda_{n}>0, \quad \lambda_{1} \geq \lambda_{2} \geq \ldots
$$

и ряд $\sum_{n=1}^{\infty} \lambda_{n}$ сходится.

Поскольку все собственные значения оператора $T$ отличны от нуля, $T$ является инъективным оператором. Стало быть, на его образе $T(H) \subset H$ определен оператор

$$
T^{-1}: T(H) \rightarrow H
$$

Обозначим через $H_{n}$ линейную оболочку векторов $e_{1}, \ldots, e_{n}$. Легко видеть, что

$$
H_{n}=T\left(H_{n}\right) \subset T(H) .
$$

Следовательно, для любого вектора $h \in H_{n}$ определен вектор $T^{-1} h$, принадлежащий подпространству $H_{n}$. 
Зададим на пространстве $H$ гауссову меру $\mu_{T}$ с корреляционным оператором $T$ и нулевым средним. Другими словами, зададим гауссову меру $\mu_{T}$, определяемую соотношениями

$$
\int_{H}(a, h) \mu_{T}(d h)=0, \quad(T a, b)=\int_{H}(a, h)(b, h) \mu_{T}(d h)
$$

для любых $a, b \in H$, где ( , ) - скалярное произведение в $H$.

Тогда для произвольной непрерывной функции $f: H \rightarrow \mathbf{C}$, удовлетворяющей услоВИю

$$
|f(h)| \leq C \exp \left(\varepsilon\|h\|^{2}\right)
$$

где $0<\varepsilon<\frac{1}{2} \lambda_{1}^{-1}$, имеет место равенство

$$
\int_{H} f(h) \mu_{T}(d h)=\lim _{n \rightarrow \infty} \frac{\int_{H_{n}} f(h) e^{-\frac{1}{2}\left(T^{-1} h, h\right)} d h}{\int_{H_{n}} e^{-\frac{1}{2}\left(T^{-1} h, h\right)} d h} .
$$

В этом выражении $d h$ - мера Лебега в конечномерном пространстве $H_{n}$. Заметим, что равенство (4) однозначно определяет меру $\mu_{T}[3]$.

Такое определение гауссовой меры позволяет формально записать

$$
\mu_{T}(d h)=C e^{-\frac{1}{2}\left(T^{-1} h, h\right)} d h, \quad h \in H .
$$

Приведем пример континуальных интегралов такого вида, встречаюшихся в квантовой теории поля. Начнем с построения ядерных операторов. Для этого рассмотрим ограниченную область $U \subset \mathbf{R}^{n}$. В качестве пространства $H$ возьмем $H^{p}(U)$ - соболевское пространство, полученное пополнением пространства $D(U) \subset D\left(\mathbf{R}^{n}\right)$ гладких функций на $U$ по скалярному произведению

$$
\left(\phi_{1}(x), \phi_{2}(x)\right)=\int_{U}\left(-\Delta+m^{2}\right)^{p} \phi_{1}(x) \phi_{2}(x) d x \quad(p>0),
$$

$\Delta=\frac{\partial^{2}}{\partial x_{1}^{2}}+\cdots+\frac{\partial^{2}}{\partial x_{n}^{2}}-$ стандартньй оператор Лапласа на $\mathbf{R}^{n}$, который задает самосопряженный оператор $\left(-\Delta+m^{2}\right)$ в $L_{2}\left(\mathbf{R}^{n}\right)$. В качестве оператора $T$ выберем сужение непрерывного оператора $\left(-\Delta+m^{2}\right)^{-p}$ на пространство $H^{p}(U) \subset L_{2}\left(\mathbf{R}^{n}\right)$. Для вьполнения условия ядерности оператора $T=\left(-\Delta+m^{2}\right)^{-p}$ положим $p>\frac{n}{2}$.

Рассмотрим теперь функциональный интеграл вида

$$
\int_{H^{p}(U)} \exp \left\{-S_{0}[\phi]-S_{\text {int }}[g, \phi]-S_{J}[\phi]\right\} d \phi
$$

здесь

$$
S_{0}=\int_{U}\left(\left(-\Delta+m^{2}\right)^{p} \phi(x)\right) \phi(x) d x
$$


Заметим, что в абстрактных обозначениях $S_{0}[\phi]$ есть не что иное, как $\left(T^{-1} \phi, \phi\right)$.

Слагаемые $S_{\mathrm{int}}[g, \phi]$ и $S_{J}[\phi]$ имеют вид

$$
S_{\mathrm{int}}[g, \phi]=g \int_{U}(\phi(x))^{4} d x, \quad S_{J}[\phi]=\int_{U} J(x) \phi(x) d x .
$$

Из теоремы Соболева о вложении $H^{p}(U) \rightarrow C_{0}(U)$ следует, что функция $S_{\mathrm{int}}$ на пространстве $H^{p}(U)$ непрерывна. Также очевидна и непрерывность $S_{J}$.

Пользуясь формулой (5), перепишем интеграл (7) как

$$
I(g)=\int_{H^{p}(U)} \exp \left\{-S_{\mathrm{int}}[g, \phi]-S_{J}[\phi]\right\} \mu_{T}(d \phi) .
$$

При тех же условиях на полевые функции $\phi$ определены интегралы (1), которые также можно переписать в виде

$$
\int_{H^{p}(U)} \phi\left(x_{1}\right) \ldots \phi\left(x_{n}\right) \exp \left\{-S_{\mathrm{int}}[g, \phi]\right\} \mu_{T}(d \phi) .
$$

Итак, в евклидовой квантовой теории поля возникает задача вычисления континуального интеграла

$$
\int_{H} F(h) e^{-g P(h)} \mu_{T}(d h),
$$

где $P(h)$ - некоторый полином, удовлетворяющий неравенству

$$
P(h) \geq \text { const }\|h\|^{2-\varepsilon} \quad(\varepsilon>0),
$$

и $F(h)$ - такая аналитическая функция на $H$, что

$$
|F(h)| \leq \text { const } \exp \left(\|h\|^{2-\varepsilon}\right) \quad(\varepsilon>0) .
$$

3. Основной метод вычисления континуальных интегралов состоит в сведении их к интегралам вида

$$
\int_{H} u_{n}(h) \mu_{T}(d h),
$$

где $u_{n}(h)$ - однородные непрерывные полиномы по $h \in H$. При этом значения интегралов (15) находятся с помошью теоремы Вика $[4,5]$.

Рассмотрим класс $\mathcal{F}(H)$ аналитических функций $f(h)$, удовлетворяюших условиям:

1)

$$
|f(h)| \leq \exp \left(C\|h\|^{\alpha}\right), \quad 0<\alpha<2,
$$

2) для любого $n>0 f^{n}(h)$ разлагается в ряд по непрерывным однородным полиномам:

$$
f^{n}(h)=\sum_{k=0}^{\infty} q_{k}(h),
$$


64 МЕТОД ПРИБЛИЖЕННОГО ВЫЧИСЛЕНИЯ КОНТИНУАЛЬНЫХ ИНТЕГРАЛОВ. ІІ

таким что

$$
\left|q_{k}(h)\right| \leq \frac{1}{k !} C_{n}^{k}\|h\|^{k \alpha}
$$

Тогда, если $f(h) \in \mathcal{F}(H)$, то для любого ядерного оператора $T$ и любого однородного непрерывного полинома $\mathcal{P}(h)$ из оценки

$$
\left|f^{n}(h)\right| \leq \exp \left(C n\|h\|^{\alpha}\right), \quad 0<\alpha<2
$$

следует, что интеграл

$$
\int_{H} \mathcal{P}(h) f^{n}(h) \mu_{T}(d h)
$$

существует.

Отметим, что нахождение этого интеграла сводится, таким образом, к вычислению интеграла (15), поскольку в силу (18)

$$
\int_{H} \mathcal{P}(h) f^{n}(h) \mu_{T}(d h)=\sum_{k=0}^{\infty} \int_{H} \mathcal{P}(h) q_{k}(h) \mu_{T}(d h) .
$$

Этим свойством мы воспользуемся в дальнейшем. Условия (16)-(18) выполняются, например, для функции

$$
f(h)=e^{i(h, y)},
$$

где $y$ - фиксированный элемент в $H$. В этом случае интеграл (20) задает преобразование Фурье гауссовой меры

$$
\widetilde{\mu}_{T}(y)=\int_{H} e^{i(h, y)} \mu_{T}(d h)=\exp \left(-\frac{1}{2}(T y, y)\right) .
$$

4. Рассмотрим теперь построение алгоритма вычисления с произвольной точностью интеграла вида

$$
\int_{H} \mathcal{P}(h) e^{-g P(h)} \mu_{T}(d h)
$$

$\mathcal{P}(h)$ - непрерывньй однородный полином. Мы покажем, что при этом возникает новая теория возмушений со сходяшимися рядами.

Пусть $P \in \mathcal{F}(h)$ - неотрицательная функция, удовлетворяюшая оценке

$$
|P(h)| \leq C_{1}(1+\|h\|)^{4}, \quad C_{1} \geq 0 .
$$

В частности, в качестве $P(h)$ можно взять непрерывный неотрицательно определенный полином четвертого порядка.

Обозначим

$$
Q(h)=P^{\frac{1}{4}}(h) .
$$


Введем функцию

$$
\widetilde{\varphi}(\rho)=\frac{1}{2 \pi} \int_{-\infty}^{+\infty} e^{-i \rho r} e^{-r^{4}} d r
$$

которая при больших значениях $|\rho|$ удовлетворяет условию [6]

$$
|\widetilde{\varphi}(\rho)| \leq C \exp \left(-|\rho|^{1+\frac{1}{4}}\right) .
$$

Тогда

$$
e^{-g P(h)}=\int_{-\infty}^{+\infty} \exp \left\{i g^{\frac{1}{4}} \rho Q(h)\right\} d \rho .
$$

Таким образом, интеграл (23) представляется в виде

$$
I(g)=\int_{H}\left(\int_{-\infty}^{+\infty} \widetilde{\varphi}(\rho) \exp \left(i g^{\frac{1}{4}} \rho Q(h)\right) d \rho\right) \mathcal{P}(h) \mu_{T}(d h) .
$$

Интеграл

$$
\int_{-\infty}^{+\infty} \tilde{\varphi}(\rho) d \rho
$$

конечен. Поэтому применима теорема Фубини, и интеграл (29) равен

$$
I(g)=\int_{-\infty}^{+\infty} \tilde{\varphi}(\rho)\left(\int_{H} \exp \left(i g^{\frac{1}{4}} \rho Q(h)\right) \mathcal{P}(h) \mu_{T}(d h)\right) d \rho .
$$

Запишем интеграл по $\rho$ как

$$
\begin{aligned}
I(g) & =\lim _{R \rightarrow+\infty} J(g, R), \\
J(g, R) & =\int_{-R}^{+R} \widetilde{\varphi}(\rho)\left(\int_{H} \exp \left(i g^{\frac{1}{4}} \rho Q(h)\right) \mathcal{P}(h) \mu_{T}(d h)\right) d \rho .
\end{aligned}
$$

Далее, так же как и в работе [1], вместо интеграла $I(g)$ мы будем рассматривать интеграл $J(g, R)$, отличающийся от $I(g)$ тем, что интегрирование по вспомогательному параметру $\rho$ проводится в конечных пределах. Это позволяет доказать возможность изменения порядка интегрирования и суммирования в формуле (33), но это и вносит зависимость от дополнительного параметра $R$. При этом нетрудно убедиться, что семейство интегралов $J(g, R)$ приближает значение $I(g)$ со сколь угодно высокой точностью $(\sim \exp (-R))$.

Разлагая экспоненту в подынтегральном выражении формулы (32) в ряд и меняя порядок интегрирования и суммирования, получим

$$
\begin{aligned}
J(g, R) & =\int_{-R}^{+R} \int_{H} \widetilde{\varphi}(\rho) \sum_{n=0}^{\infty} \frac{i^{n}}{n !} g^{\frac{n}{4}} \rho^{n} Q^{n}(h) \mathcal{P}(h) \mu_{T}(d h) d \rho= \\
& =\sum_{n=0}^{\infty} \frac{i^{n}}{n !} g^{\frac{n}{4}} \int_{-R}^{+R} \widetilde{\varphi}(\rho) \rho^{n} d \rho \int_{H} Q^{n}(h) \mathcal{P}(h) \mu_{T}(d h) .
\end{aligned}
$$

3 Теоретическая и математическая физика, т. 109, № 1, 1996 г. 
Для доказательства законности перестановки операций интегрирования и суммирования запишем $J(g, R)$ в виде

$$
\int_{-R}^{+R} \int_{H}\left(\sum_{n=0}^{\infty} \frac{i^{n}}{n !} g^{\frac{n}{4}} \tilde{\varphi}(\rho) \rho^{n} Q^{n}(h) e^{-\delta\|h\|^{2}}\right) e^{\delta\|h\|^{2}} \mathcal{P}(h) \mu_{T}(d h) d \rho,
$$

где $0<\delta<\frac{1}{2} \lambda_{1}^{-1}$.

Заметим теперь, что справедливы следующие оценки:

$$
\begin{gathered}
\left|\widetilde{\varphi}(\rho) \rho^{n}\right| \leq R^{n}, \quad \int_{-R}^{+R}\left|\widetilde{\varphi}(\rho) \rho^{n}\right| d \rho \leq \frac{1}{n+1} R^{n+1} \\
\left|Q^{n}(h) e^{-\delta\|h\|^{2}}\right| \leq\left(C_{1}(1+\|h\|)^{4}\right)^{\frac{n}{4}} e^{-\delta\|h\|^{2}} \leq C_{2}^{n} n^{\frac{n}{2}} e^{-\frac{n}{2}}
\end{gathered}
$$

(Для получения последнего неравенства мы использовали максимальное значение функции $\left(1+t^{4}\right)^{\frac{n}{4}} \exp \left(-\delta t^{2}\right)$.) Так как интеграл

$$
\int_{H} \exp \left(\delta\|h\|^{2}\right) \mathcal{P}(h) \mu_{T}(d h)
$$

конечен, то

$$
\int_{H}\left|Q^{n}(h) e^{-\delta\|h\|^{2}}\right| e^{\delta\|h\|^{2}} \mathcal{P}(h) \mu_{T}(d h) \leq C_{3}^{n} n^{\frac{n}{2}} .
$$

Поскольку

$$
\left|\frac{1}{n !} g^{\frac{n}{4}} \widetilde{\varphi}(\rho) \rho^{n} Q^{n}(h) e^{-\delta\|h\|^{2}}\right| \leq C_{4}^{n} g^{\frac{n}{4}} \frac{1}{\Gamma\left(\frac{n}{2}\right)} R^{n}
$$

ряд в подынтегральном выражении формулы (34) сходится равномерно. Кроме того, с учетом неравенств (35), (37) получаем оценку

$$
\int_{-R}^{+R} \int_{H}\left|\frac{1}{n !} g^{\frac{n}{4}} \widetilde{\varphi}(\rho) \rho^{n} Q^{n}(h) e^{-\delta\|h\|^{2}} \mathcal{P}\right| \mu_{T}(d h) d \rho \leq C^{n} g^{\frac{n}{4}} \frac{1}{(n+1) \Gamma\left(\frac{n}{2}\right)} R^{n+1} .
$$

Выполнение неравенств (38), (39) доказывает справедливость формулы (33).

Таким образом, интеграл $J(g, R)$ раскладывается в абсолютно сходяшийся ряд:

$$
J(g, R)=\sum_{n=0}^{\infty} \frac{1}{n !} g^{\frac{n}{4}} A_{n}(R) \int_{H} Q^{n}(h) \mathcal{P}(h) \mu_{T}(d h)
$$

Коэффициенты

$$
A_{n}(R)=i^{n} \int_{-R}^{+R} \tilde{\varphi}(\rho) \rho^{n} d \rho
$$


те же самые, что и для обычного конечномерного интеграла (см. [1]). Они отличны от нуля только при четных $n$. Поэтому абсолютно сходяшийся ряд (41) можно записать в виде

$$
\begin{aligned}
J(g, R)= & \sum_{k=0}^{\infty} \frac{1}{(4 k) !} g^{k} A_{4 k}(R) \int_{H}(P(h))^{k} \mathcal{P}(h) \mu_{T}(d h)+ \\
& +\sum_{k=0}^{\infty} \frac{1}{(4 k+2) !} g^{k+\frac{1}{2}} A_{4 k+2}(R) \int_{H}(P(h))^{k+\frac{1}{2}} \mathcal{P}(h) \mu_{T}(d h) .
\end{aligned}
$$

Обратим внимание на то, что при обычном разложениии интеграла (23) в расходящийся степенной ряд по $g$ второй часть формулы (42) отсутствует, так как

$$
\lim _{R \rightarrow+\infty} A_{4 k}(R)=\frac{1}{\pi} \frac{(4 k) !}{k !}, \quad \lim _{R \rightarrow+\infty} A_{4 k+2}(R)=0 .
$$

Итак, формула (42) определяет новую теорию возмущений, ряды которой, в отличие от асимптотических разложений стандартной теории возмушений, сходятся. Фактически формула (42) представляет собой разложение интеграла $J(g, R)$ по степеням параметра $g^{\frac{1}{2}}$.

Континуальные интегралы в первом из рядов (42) повторяют интегралы стандартной теории возмущений и вычисляются обычным способом. Что же касается континуальных интегралов из второго ряда

$$
\int_{H} \mathcal{P}(h) P^{k+\frac{1}{2}}(h) \mu_{T}(d h),
$$

то в этом случае можно поступить следуюшим образом.

Запишем формулу (44) так:

$$
\int_{H} \mathcal{P}(h) P^{k+\frac{1}{2}}(h) e^{-\delta\|h\|^{2}} e^{\delta\|h\|^{2}} \mu_{T}(d h),
$$

где $0<\delta<\frac{1}{2} \lambda_{1}^{-1}$.

Рассмотрим функцию

$$
y=y(h)=P(h) e^{-\frac{2}{2 k+1} \delta\|h\|^{2}}, \quad h \in H .
$$

Она неотрицательна и ограничена при всех $h$. Другими словами, сушествует $\sup y(h)=y_{0}$ и $0 \leq y \leq y_{0}$. По теореме Вейерштрасса функцию $\psi(y)=y^{k+\frac{1}{2}}$, рассматриваемую на отрезке $\left[0, y_{0}\right]$, можно сколь угодно точно приблизить полиномом по $y$. В результате подынтегральное выражение будет представлено как полиномы 
по $h$, умноженные на степени $\exp \left(-\frac{2}{2 k+1} \delta\|h\|^{2}\right)$. В итоге задача сведется к вычислению интегралов следуюшего вида:

$$
\int_{H} \mathcal{P}(h) P^{l}(h) e^{-\left(\frac{2 l}{2 k+1}-1\right) \delta\|h\|^{2}} \mu_{T}(d h),
$$

которые находятся с помощью формулы (21).

Отсюда следует, что с любой точностью интеграл (45) можно приблизить вычислимым континуальным интегралом от полинома по некоторой гауссовой мере. Отметим, что выбор той или иной схемы приближения определяет конкретное обобщение стандартной диаграммной техники для новой теории возмущений.

Выбор четвертой степени в формуле (24) (или полинома четвертого порядка) был достаточно произволен. Вместо четвертой степени можно было бы взять произвольную степень $2 m$. Формулы (24)-(42) без труда обобщаются на этот случай. В итоге для этого обобщения (мы здесь явно указываем зависимость от $m$ ) получим

$$
I(m, g)=\lim _{R \rightarrow+\infty} J(m, g, R)
$$

где

$$
\begin{aligned}
J(m, g, R) & =\sum_{k=0}^{\infty} \frac{1}{(2 k) !} g^{\frac{k}{m}} A_{2 k}(m, R) \int_{H}(P(h))^{\frac{k}{m}} \mathcal{P}(h) \mu_{T}(d h), \\
A_{2 k}(m, R) & =i^{2 k} \int_{-R}^{+R} \widetilde{\varphi}(m, \rho) \rho^{2 k} d \rho \\
\widetilde{\varphi}(m, \rho) & =\frac{1}{2 \pi} \int_{-\infty}^{+\infty} e^{-i \rho r} e^{-r^{2 m}} d r .
\end{aligned}
$$

В этом случае параметром степенного разложения является $g^{\frac{1}{m}}$, и абсолютно сходящийся ряд теории возмушений для $J(m, g, R)$ эффективно разбивается на $m$ сходяшихся рядов, степени $g($ и $P(x))$ в которых имеют вид

$$
g^{\frac{1}{m}+n}, \ldots, g^{\frac{m-1}{m}+n}, g^{1+n}, \quad n=0,1, \ldots
$$

Континуалные интегралы от нецелых степеней $P(h)$ также можно с любой точностью приблизить вычислимыми континуальными интегралами от полиномов по некоторым гауссовым мерам.

Итак, мы показали, что любому допускающему строгое определение в смысле (4) континуальному интегралу в евклидовой квантовой теории поля можно поставить в соответствие абсолютно сходяшийся ряд, каждый член которого представляет собой континуальный интеграл от полинома по некоторой гауссовой мере.

Таким образом, в силу абсолютной сходимости ряда задача вычисления с произвольной точностью исходного континуального интеграла сводится к вычислению конечного числа квазигауссовых континуальных интегралов. Требование, чтобы оператор $T$ был ядерным, выделяет, в сушности, лишь конечные теории.

Работа выполнена при частичной поддержке РФФИ. 


\section{Список литературы}

[1] В.В. Белокуров, Ю.П. Соловьев, Е.Т. Шавзулидзе. ТМФ. 1996. Т. 109. № 1. С. 51-59.

[2] Large-order behaviour of perturbation theory. Eds J.-C. le Guillou, J. Zinn-Justin. Amsterdam: North-Holland, 1990.

[3] Х.-С. Го. Гауссовские меры в банаховых пространствах. М.: Мир, 1979.

[4] Б. Саймон. Модель $P(\varphi)_{2}$ эвклидовой квантовой теории поля. М.: Мир, 1976.

[5] Джс. Глимм, А. Джаффе. Математические методы квантовой физики. Подход с использованием функциональных интегралов. М.: Мир, 1984.

[6] О.Г. Смолянов, Е.Т. Шавгулидзе. Континуальные интегралы. М.: Изд-во МГУ, 1990.

Поступила в редакцию 11.IX.1995 г.

\section{V. Belokurov, Yu. P. Solov'ev, E. T. Shavgulidze \\ METHOD OF APPROXIMATE CALCULATING PATH INTEGRALS \\ BY USING PERTURBATION THEORY WITH CONVERGENT SERIES. II. EUCLIDEAN QUANTUM FIELD THEORY}

We establish a method of approximate calculating path integrals, proposed in our previous paper, for the case where the Gaussian measure is given by an operator of trace class. 